\title{
An unusual cause of terminal hematuria in a child: Eosinophilic cystitis
}

\author{
Elif Bahat Özdoğan, MD; ${ }^{*}$ Seçil Arslansoyu Çamlar, MD;* Sevcan Bilen, MD; ${ }_{;}^{\dagger}$ Mustafa İmamoğlu, MD; ; \\ Şükran Tıraş, MD, ${ }^{\dagger}$ Ayşegül Cansu, MD; ${ }^{*}$ Yavuz Özoran, $M D^{ \pm}$
}

*Department of Pediatrics, Division of Pediatric Nephrology, Faculity of Medicine, Karadeniz Tecnical University, Trabzon, Turkey; 'Department of Pediatrics, Faculity of Medicine, Karadeniz Tecnical University, Trabzon, Turkey; ${ }^{\circledR}$ Department of Pediatric Surgery, Faculity of Medicine, Karadeniz Tecnical University, Trabzon, Turkey; ${ }^{*}$ Department of Radiology, Faculity of Medicine, Karadeniz Tecnical University, Trabzon, Turkey; :Department of Medical Pathology, Faculity of Medicine, Faculity of Medicine, Karadeniz Tecnical University, Trabzon, Turkey

Cite as: Can Urol Assoc J 2014;8(11-12):e867-71. http://dx.doi.org/10.5489/cuaj.2173 Published online November 24, 2014.

\section{Abstract}

Eosinophilic cystitis is a rare inflammatory disease of the bladder; it rarely occurs in children. Patients typically show irritative urination symptoms frequently, with a possible need for urgency, alongside dysuria, gross haematuria, suprapubic pain and painful urination. Sometimes bladder mass accumulation with the possibility of malignancy is also observed. We present an 8-year-old male patient who gained admission for terminal hematuria and discuss the management of eosinophilic cystitis.

\section{Introduction}

Terminal hematuria occurs at the end of the urinary tract and may have a prostatic, bladder, or trigonal cause. ${ }^{1}$ Gross hematuria in children is rare and is often associated with benign conditions, involving an infection or a urolithiasis. ${ }^{2,3}$ Eosinophilic cystitis (EC) is a rare inflammatory disease of the bladder with unknown etiology, characterized by numerous eosinophilic infiltrations of the bladder. ${ }^{4}$ Patients with eosinophilic cystitis show irritative urination symptoms frequently, with a possible need for urgency, alongside dysuria, gross hematuria, suprapubic pain and painful urination. Sometimes bladder mass accumulation with the possibility of malignancy is also observed. We present an 8-year-old male patient who gained admission for terminal hematuria and discuss the management of EC.

\section{Case report}

An 8-year-old male was admitted to hospital with a complaint of acute onset bleeding post-urination. He had frequent dysuria, with no fever or abdominal pain. One month before his admission, the patient had been admitted to another hospital with complaints of frequent terminal hematuria and antibiotics were prescribed for the treatment of suspected urinary tract infection. There was no history of any allergy or external trauma. Vital signs showed an axillary temperature of $37{ }^{\circ} \mathrm{C}$, heart rate of $88 / \mathrm{s}$, and a blood pressure of 90/60 mmHg. Physical examination was normal.

Hematologic laboratory findings were: hemoglobin density of $12.4 \mathrm{~g} / \mathrm{dL}$; white blood cell count of $5800 / \mathrm{mm}^{3}$, with $64 \%$ neutrophils, $72 \%$ lymphocytes, $4 \%$ monocytes and $5 \%$ eosinophils; platelet count of $377000 / \mathrm{mm}^{3}$; blood urea nitrogen density of $12 \mathrm{mg} / \mathrm{dL}$, creatinine density of $0.44 \mathrm{mg} /$ $\mathrm{dL}$, calcium, phosphate, uric acid, and alkaline phosphatase levels with erythrocyte sedimentation rate (ESR) of $21 \mathrm{~mm} / \mathrm{h}$. Furthermore, C-reactive protein level was normal $(0.06$ $\mathrm{mg} / \mathrm{L})$. Complement C3 density was $129 \mathrm{mg} / \mathrm{dL}$ (N:90-180), immunoglobulin $\mathrm{E}$ (Ig E) $256 \mathrm{IU} / \mathrm{mL}$ (N:1.35-165), immunoglobulin A (IgA) $119 \mathrm{mg} / \mathrm{dL}(\mathrm{N}: 1-250)$, antistreptolysin $\mathrm{O}$ antibody $438 \mathrm{IU} / \mathrm{mL}$ (normal $<200 \mathrm{IU} / \mathrm{mL}$ ), and finally the absolute eosinophil count was $300 / \mathrm{mm}^{3}$. Allergy tests failed to identify any specific antigen. In the urine sample, urine specific gravity was 1020 , with no present protein; urine nitrite was negative, though many erythrocytes were observed. The urine sediment on microscope demonstrated numerous eumorphologic erytrocyte counts per field. Urine eosinophil percentage was 22\% (with normal counts showing $<3 \%$ ). Spot urine calcium to creatinine ratio was 0.02. Urine culture was sterile.

Ultrasonography analysis revealed diffuse enlargement of the bladder wall. Magnetic resonance imaging (MRI) of the lower abdomen revealed $11 \mathrm{~mm}$ diffuse thickening in all segments of the bladder wall (Fig. 1), which also had been confirmed by the urinary ultrasonography analysis. Cystoscopy was conducted to assess for suspected eosinophilia and eosiphiluria. Cystoscopic examination showed oedematous mucosal erythema with no polypoid lesion. Microscopic examination of the urinary bladder revealed chronic inflammation, including a prominent eosinophilia involving the mucosa and the full thickness of the lamina 
propria, demonstrating the standard case with eosinophilic cystitis (Fig. 2). Antihistamines, klaritromicine and nonsteroidal antiinflammatory treatment was started. He was followed up for 4 months. After the initial treatment, bladder wall thickness decreased from $11 \mathrm{~mm}$ to $4 \mathrm{~mm}$ and there was no microscopic hematuria.

\section{Discussion}

Hematuria is a frequently encountered symptom that has a broad differential diagnosis, ranging from urinary system infection to bladder cancer. Terminal hematuria is a pathology encountered mostly as a result of inflammation of bulbar urethra and bladder neck. EC may be mimicking bladder cancer because of mass lesion in bladder. Patients with severe cases may need radical cystectomy. ${ }^{5}$

Terminal hematuria also can be seen in both EC and bladder cancer. Therefore, the differential diagnosis is very important. Microscopic hematuria frequently is benign, but macroscopic hematuria may indicate severe disease (Fig. 3).

$\mathrm{EC}$ is a rare inflammatory disease of the bladder, first described in 1960 by Brown. ${ }^{1}$ It has an unknown etiology, though it is characterized by numerous eosinophilic infiltrations of the bladder. ${ }^{1}$ Patients with EC show irritative urination symptoms frequently, with possible urgency, alongside dysuria, gross hematuria, suprapubic pain and painful urination. Sometimes bladder mass accumulation with the possibility of malignancy is also observed. Some patients have microscopic hematuria, urinary retention, and nocturia. EC rarely occurs during chilhood; less than 75 pediatric cases have been described and there is more morbidity in boys. ${ }^{2,3}$ There is no genetic predisposition.

Vesical injury, chronic vesical irritation, bladder surgery, food and drug allergies, tuberculosis and malignancies can be causal factors of EC; however, etiologic agents cannot easily be identified. ${ }^{4}$ Intravesical mitomycin, thiotepa and dimethyl sulfoxide have been reported as possible causative

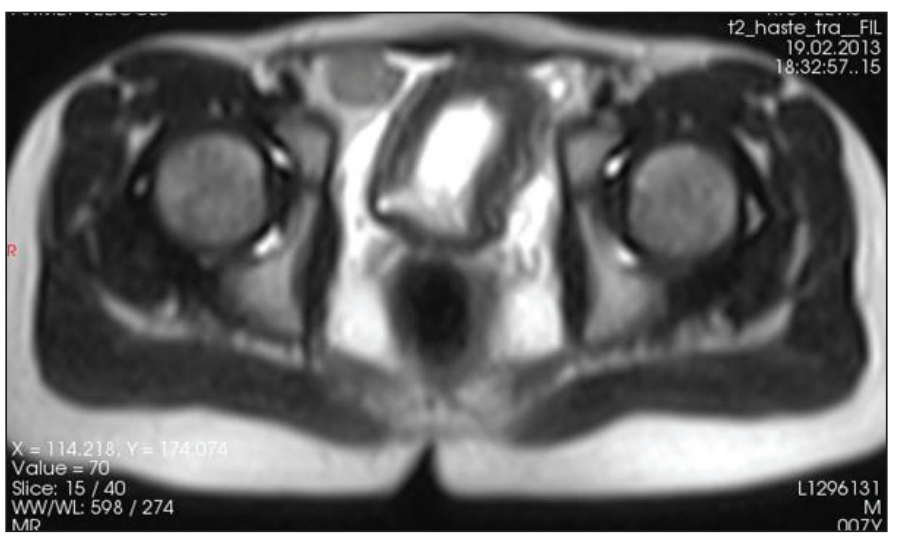

Fig. 1. Microscopic examination of the urinary bladder. Chronic inflammation (prominent eosinophilia) and full thickness of the lamina propria. agents. ${ }^{6,7}$ Food allergens and parasites, such as Toxocara canis and Echinococcus, have been used to clarify the onset of EC. ${ }^{8}$ Conditions that have been associated with EC include Glanzmann's thrombasthenia, eosinophilic gastroenteritis, bronchial asthma, and coeliac disease. ${ }^{9}$ Although peripheral eosinophilia and eosinophilia were highly suggestive of an underlying allergic cause, no specific cause was identified in this study.

The pathophysiology is unclear. EC can be related to a disorder of the immunoregulatory system due to exposure to a foreign protein substance acting as an antigenic stimulus. This can cause an immunoglobulin E-mediated release of eosinophilic chemotactic factor which would ultimately lead to the release of lysosomal enzymes and cytokines. ${ }^{3}$

With our patient, physical examination revealed suprapubic tenderness, flank tenderness, or a mass aggregation anterior to the rectum. Urinalysis showed proteinuria, pyuria and microscopic hematuria. Serum eosinophilia was observed. Positive urine culture had been reported; however, this was negative in our case. Mild peripheral blood eosinophilia had also been reported, with rare occurence as hypereosinophilic syndrome. ${ }^{8}$ EC has its own diagnostic management (Fig. 4). ${ }^{5}$

Ultrasonography and MRI results showed prominent thickening of the bladder wall. Cystoscopic abnormalities in EC ranged from mucosal erythema to elevated, velvety, polypoid, oedematous, clearly aggregating, and invasive-appearing masses. Bladder biopsy was required for diagnosis. Histologically, the lamina propria is oedematous, containing a mixed inflammatory infiltrate in which eosinophils are prominent. ${ }^{5}$ Occasionally, edema causes bladder outlet obstruction and urinary retention. ${ }^{3}$ Eosinophilic infiltrate was scattered throughout the lamina propria, and muscularis on bladder biopsy showed pathognomony for eosinophilic cystitis (Fig. 2). Mast cells, plasma cells, and giant cells were observed.

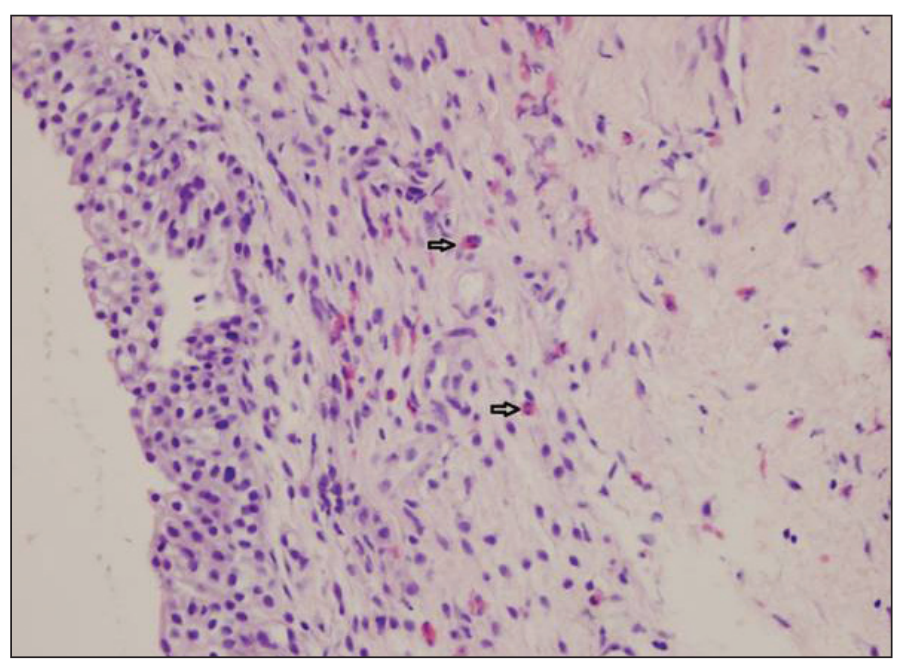

Fig. 2. Magnetic resonance imaging of the lower abdomen revealed diffuse thickening in all segments of the bladder wall. 


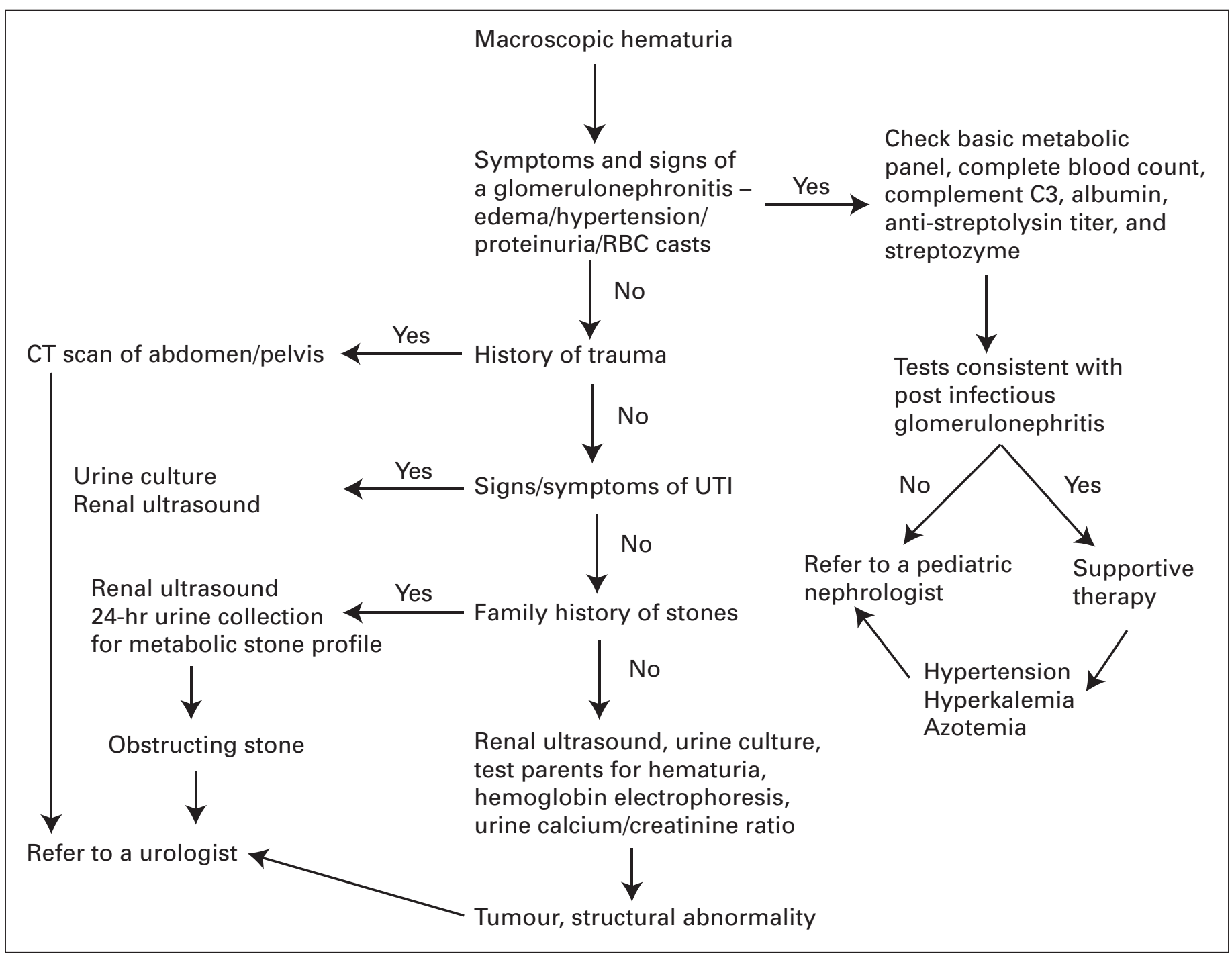

Fig. 3. Macroscopic hematuria algorithm. RBC: red blood count; UTI: urinary tract infection; CT: computed tomography.

Conservative medical management is advised. Nonspecific medical therapy involving non-steroidal anti-inflammatory agents, steroids, antihistamines, cyclosporine A, or montelukast could be used. ${ }^{2,4,10}$ Medical treatment was initiated with non-steroidal anti-inflammatory ibuprofen for $10 \mathrm{mg} / \mathrm{kg}$ in 3 doses, and antihistaminic desloratadine for $5 \mathrm{mg} /$ day. Desloratadine is an $\mathrm{H} 2$ class antihistamine that stabilizes mast cells and possibly impairs eosinophilic migration to target organs. Hematuria showed complete resolve and bladder thickness decreased to $4 \mathrm{~mm}$ over a 4-week period since the initiation of medical therapy. Therapy itself continued for 6 months. One year later, the patient was still asymptomatic; follow-up was still needed as recurrence may ocur. ${ }^{11}$ Surgery involving cystectomy or urinary diversion had been reported, but should only be considered after exhausting all other therapeutic options. Eosinophilic gastroenteritis, vesicoureteral reflux, hematuria requiring blood transfusion or salvage cystectomy, eosinophilic ureteritis, bladder rupture, and renal insufficiency are rare complications associated with EC. Sparks and colleagues ${ }^{3}$ reported on 4 cases with EC. All patients were diagnosed via cystourethroscopy with biopsy. Patients were treated with a combination of steroids, antihistamines, and antibiotics. The authors concluded that EC presented with many different symptoms and diagnosis requires a high index of suspicion.

Although very rare during chilhood, EC should also be considered in patients with terminal hematuria which is responsive to medical therapy following nonsteroidal medications, as is the case with our patient. It also is often benign, despite the symptoms of bladder mass possibly directing to malignancy. EC can mimick bladder cancer because of the accompanying bladder mass. Dysuria, frequent urination, suprapubic pain or urinary retention due to obstruction and pelvic fullness may be seen in both EC and bladder cancer. 


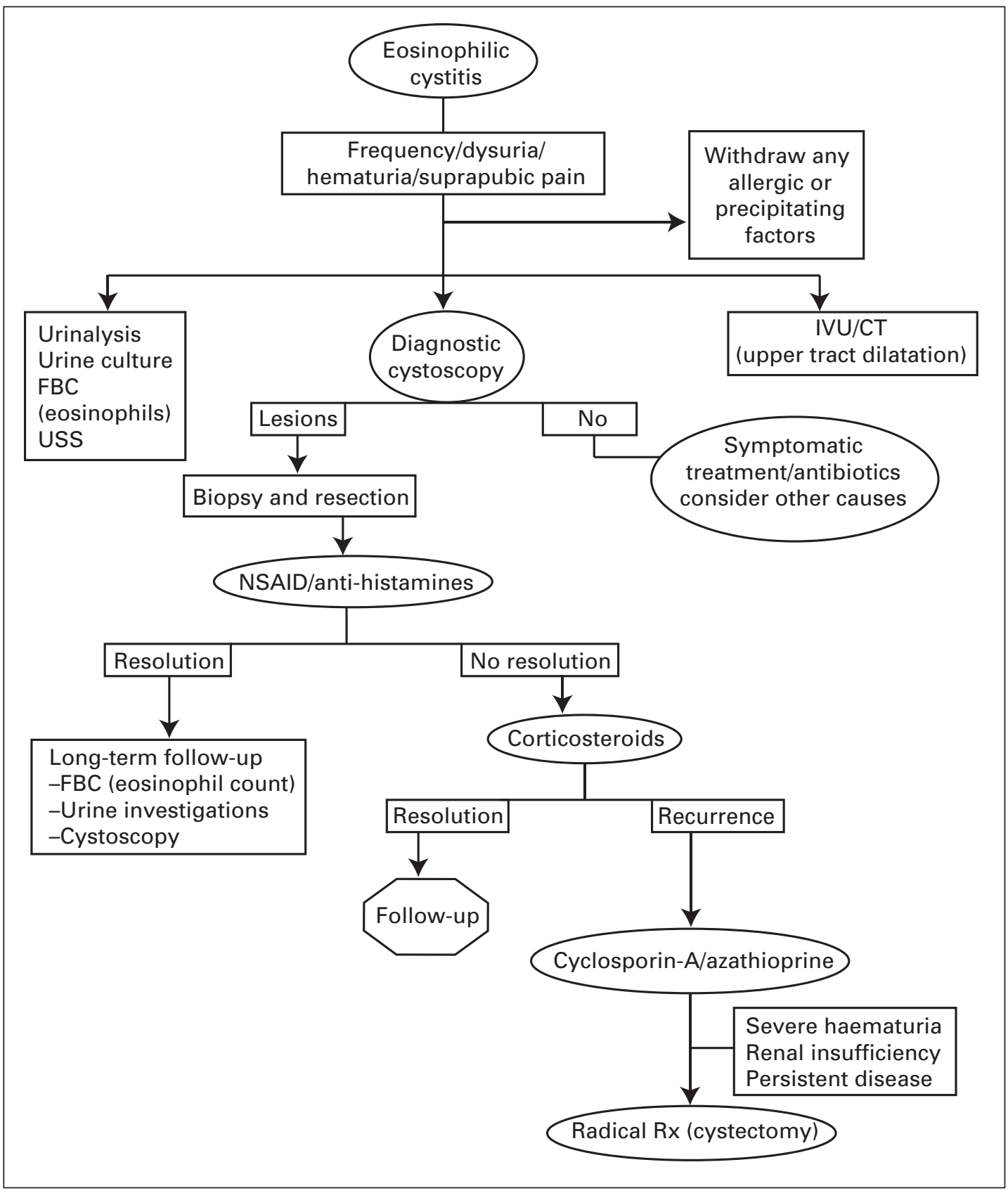

Fig. 4. Algorithm of eosinophilic cystitis.

If EC causes a fibrosis in the bladder and a dilatation in the urinary system, it may present as a bladder mass mimicking bladder cancer. ${ }^{12}$

\section{Conclusion}

When a patient presents with macroscopic hematuria and if there is a mass lesion in the bladder, cystoscopy and biopsy should be performed in the differential diagnosis. Histological examination is recommended to establish the diagnosis.

Competıng interests: Dr. Özdoğan, Dr. Tiraș, Dr. Çamlar, Dr. İmamoğlu, Dr. Cansu, Dr. Özoran and Dr. Bilen declare no competing financial or personal interests.

This paper has been peer-reviewed. 


\section{References}

1. Brown EW. Eosinophilic granuloma of the bladder. J Urol 1960;83:665-8.

2. Pomeranz A, Eliakim A, Uziel Y, et al. Eosinophilic cyctitis in a 4-year old boy: Successful long term treatment with cyclosporin A. Pediatrics 2001;108:e1 13. http://dx.doi.org/10.1542/peds. 108.6.el13

3. Sparks $S$, Kaplan A, Decambe M, et al. Eosinophilic cystitis in the pediatric population: A case series and review of the literature. J Pediatr Urol 2013; 9 (6 P† A):738-44.

4. Sterrett $S$, Morton J, Perry D, et al. Eosinophilic cystitis:successful long-term treatment with montelukast sodium. Urology 2006;67:423.e19-423.e21.

5. Teegavarapu PS, Sahai A, Chandra A, et al. Eosinophilic cystitis and its management. Int I Clin Pract 2005;59:356-60. http://dx.doi.org/10.1111/j.1742-1241.2004.00421.x

6. Choe JM, Kirkemo AK, Sirls LT. Intravesical thiotepa-induced eosinophilic cystitis Urology 1995;46:729-31. http://dx.doi.org/10.1016/S0090-4295(99)80312-4

7. Inglis JA, Tolley DA, Grigor KM. Allergy to mitomycin C complicating topical administration for urothelial cancer. Br J Urol 1987;59:547-9. http://dx.doi.org/10.1111/j.1464-410X.1987.tb04874.x
8. Samuel M, Misra D, Larcher V, et al. Schistosoma haematobium infection in children in Britain. BJU Int 2000;85:316-8. http://dx.doi.org/10.1046/i.1464-410x.2000.00477.x

9. Botma JP, Burger EG, de Kock ML. Eosinophilic cystitis associated with Glanzmann's thrombasthenia. A case report. S Afr Med J 1987;71:533-4.

10. Motzkin D. Nonsteroidal anti-inflammatory drugs inthe treatment of eosinophilic cystitis. J Urol 1990;144:1464-6.

11. Van den Ouden D. Diagnosis and management of eosinophilic cystitis: A pooled analysis of 135 cases. Eur Urol 2000;37:386-94. http://dx.doi.org/10.1159/000020183

12. Manimaran D, Karthikeyan TM, Sreenivasulu M, et al. Eosinophilic cystitis mimicking bladder tumour - a rare case report. J Clin Diagn Res 2013;7:2282-3.

Correspondence: Dr. Elif Bahat Özdoğan, Karadeniz Tecnical University, Faculty of Medicine, Department of Pediatrics, Division of Pediatric Nephrology, 61080 Trabzon, Turkey; elifbahat@ yahoo.com 